\title{
Characterisation of the spectrum of demyelinating disease in Western Australia
}

J-S Wu, M-N Zhang, W M Carroll, et al.

J Neurol Neurosurg Psychiatry 2008 79: 1022-1026 originally published online March 20, 2008

doi: 10.1136/jnnp.2007.131177

Updated information and services can be found at:

http://jnnp.bmj.com/content/79/9/1022.full.html

Topic collections Articles on similar topics can be found in the following collections

$$
\begin{aligned}
& \text { Immunology (including allergy) (44045 articles) } \\
& \text { Cranial nerves ( } 2315 \text { articles) } \\
& \text { Drugs: CNS (not psychiatric) (8422 articles) } \\
& \text { Infection (neurology) (7849 articles) } \\
& \text { Multiple sclerosis (1523 articles) } \\
& \text { Neuromuscular disease ( } 8373 \text { articles) } \\
& \text { Peripheral nerve disease (3623 articles) } \\
& \text { Spinal cord (4195 articles) } \\
& \text { Ophthalmology (12902 articles) }
\end{aligned}
$$

Notes

To order reprints of this article go to:

http://jnnp.bmj.com/cgi/reprintform

To subscribe to Journal of Neurology, Neurosurgery \& Psychiatry go to: http://jnnp.bmj.com/subscriptions 


\title{
Characterisation of the spectrum of demyelinating disease in Western Australia
}

\author{
J-S Wu, ${ }^{1}$ M-N Zhang, ${ }^{2}$ W M Carroll, ${ }^{1}$ A G Kermode ${ }^{1}$
}

${ }^{1}$ Australian Neuromuscular Research Institute, Sir Charles Gairdner Hospital and Centre for Neuromuscular and Neurological Disorders, University of Western Australia, Perth, WA, Australia; ${ }^{2}$ First Hospital of Shanxi Medical University, Taiyuan, China

Correspondence to:

Dr Allan G Kermode, Australian

Neuromuscular Research

Institute, Sir Charles Gairdner

Hospital and Centre for

Neuromuscular and Neurological

Disorders, University of Western

Australia Queen Elizabeth II

Medical Centre, Perth 6009,

WA, Australia; kermode@mac. com

J-SW and M-NZ contributed equally to this manuscript

Received 29 July 2007

Revised 12 January 2008

Accepted 9 February 2008

Published Online First

20 March 2008

\section{ABSTRACT}

Background: The diversity of multiple sclerosis (MS) and the nosology of the conventional form of MS (CMS), optic-spinal MS (OSMS) and neuromyelitis optica (NMO) have been subject to controversy.

Aims: The purpose of this study was to investigate whether the current Asian optic-spinal multiple sclerosis (OSMS) criteria could also apply in Western countries, and whether or not cerebrospinal fluid (CSF) and imaging features in the Western Australian patient population of demyelinating disease was similar to that found in Asia.

Methods: This study retrospectively reviewed 915 individual case notes with central nervous system demyelinating disease seen by two neurologists in Western Australia (WA). 842 cases had sufficient data to be included in the analysis. The patient population was predominantly Caucasian, representing approximately two-thirds of MS cases in WA. The mean duration of follow-up for the whole studied cohort was 12.5 years, with 136 patients (16.2\%) being followed-up for more than 20 years.

Results: The study confirmed the relatively low frequency of OSMS as a proportion of total demyelinating disease occurring in western countries, with 31 OSMS (3.7\%) cases in contrast to $703 \mathrm{CMS}$ cases (83.5\%). It is likely, however, that our retrospective classification significantly underestimated the proportion of OSMS cases when compared with prospectively classified Asian cohorts. There were 11 OSMS cases that could also be classified as NMO according to published diagnostic criteria. The remainder of the spectrum comprised clinically isolated syndromes such as 50 acute myelitis (AM, 5.9\%), 42 optic neuritis (ON, 5\%) and 16 "atypical" cases such as tumefactive MS and acute disseminated encephalomyelitis (1.9\%). The clinical characteristics of OSMS in our study were compatible with so-called Asian MS in many respects: oligoclonal bands (OCBs) were less frequent in OSMS $(29.4 \%)$ than in CMS $(66.4 \%, p=0.003)$; visual evoked potentials and spinal MRI abnormalities were more prevalent in OSMS (85\% and 92.6\%) than in CMS $(71.4 \%$ and $85 \%)$; as were long spinal cord lesions in OSMS $(22.2 \%)$ versus CMS $(3.4 \%, p, 0.001)$. Brain abnormalities were seen in $48.4 \%$ of OSMS patients and $96.2 \%$ of CMS patients $(p=0.001)$. OCBs were identified in $7 \%$ of acute myelitis, $14.3 \%$ of optic neuritis and $73.4 \%$ of primary progressive MS patients.

Conclusions: This cross-sectional study presents the full spectrum of demyelinating disease in WA, which has a stable population representing $10 \%$ of the total Australian population and suggests that the current classifications of MS, OSMS or NMO, ON and AM share many clinical and laboratory features, such as female predominance, age at onset, duration of disease and CSF investigations

(including OCBs and MRI). Moreover, characteristics of the WA population were similar to those reported in Asian patients.
Multiple sclerosis (MS) is the most common neurological disorder of young adults in western countries. MS in patients of European descent is characterised by a high frequency of oligoclonal $\mathrm{IgG}$ bands (OCBs) in the cerebrospinal fluid (CSF) and the predominant conventional form of MS (CMS), with disseminated lesions in the central nervous system (CNS) including in the cerebrum, cerebellum and brainstem. ${ }^{12}$ Clinically opticospinal multiple sclerosis (OSMS) is characterised by selective involvement of the optic nerves and spinal cord and rare involvement of other parts of the CNS. In Australia, a diagnosis of OSMS is uncommon, and in the main is probably restricted to those patients classified as having Devic's disease or neuromyelitis optica (NMO). NMO is described as a variant of MS that consists of sequential or simultaneous acute myelitis (AM) and optic neuritis $(\mathrm{ON})$, without clinical disease outside the optic nerve or the spinal cord, ${ }^{3}$ which is similar to OSMS in many respects. ${ }^{4}$ Some authors, however, have recently re-classified NMO and suggested that many do, in fact, have brain lesions, usually similar to CMS but rarely with atypical features. ${ }^{5}$ Thus, the diversity and nosology of MS-such as recurrent ON, recurrent AM, OSMS and NMO-have been topical. Whether the clinical presentation and immunological features of MS patients in western countries are distinct from Asian patients with MS remains unclear. The geographical isolation of Western Australia (WA) and relatively stable population $(\sim 2$ million) provides a unique opportunity to characterise a large cohort of patients with different clinical forms of MS.

\section{METHODS}

\section{Patients}

The individual case notes of 915 patients coded as having CNS demyelinating disease (MS, Devic's disease/syndrome, NMO, ON, AM, acute disseminated encephalomyelitis (ADEM) and post-infectious encephalitis) in Perth at Sir Charles Gairdner Hospital (SCGH) from January 1990 to March 2006 were reviewed retrospectively. Of these, sufficient clinical and diagnostic information was available on 842 patients to be included in the analysis. These patients had been assessed by two neurologists in our MS clinic (AGK and WMC) between 1995 and 2005.

We recorded clinical, CSF and MRI (1.5T or 3.0T) results at baseline. CSF analysis, visual evoked potentials (VEPs) and systemic disease evaluation (clinical and biological screening for systemic lupus erythematosus, antiphospholipid syndrome and Sjögren's syndrome) were also 
obtained at the onset of disease. CSF was evaluated by white cell count (WCC), protein level and oligoclonal bands (OCBs) using highly sensitive isoelectrofocusing. ${ }^{6}$ Brain and spinal MRI abnormalities were defined as positive if they showed typical demyelinating lesions. VEPs were recorded to wide-field, halffield and central-field stimulation with subcomponent analy$\operatorname{sis}^{7}$ and all were read by the one neurologist (WMC). Abnormalities were based primarily on P100 latency or marked P100 amplitude loss for which there was no ophthalmological cause identified.

According to the criteria of Poser et al ${ }^{9}$ and Compston et al, ${ }^{10}$ 703 CMS patients were diagnosed as having clinically definite (666 cases, $94.7 \%$ ) or probable MS (37, 5.3\%). Of these, 53 were classified as primary progressive MS (PPMS) according to the Compston diagnostic criteria. Patients with lesions confined to the optic nerve and spinal cord, with no MRI evidence of lesions in the cerebrum or cerebellum, or minor lesions not satisfying the Barkhof diagnostic criteria, were classified as having OSMS. ${ }^{11}$ As specified in current OSMS criteria, patients who met the above criteria with minor brainstem signs (eg, internuclear ophthalmoplegia) were also classified as having OSMS. Because of the nature of this study, the criteria were applied when assessed by AGK or WMC, hence OSMS numbers may be underestimated if they had progressed to develop brain lesions after their initial presentation. We also applied the NMO diagnostic criteria ${ }^{12}$ to these patients, as the diagnosis of $\mathrm{NMO}$ requires all the three absolute criteria, which are exclusively clinical (ON, AM and no demyelinating disease evident beyond optic nerve and spinal cord), and one major supportive criterion or two minor supportive criteria. Among the major supportive criteria, two refer to MRI (negative brain MRI at onset or does not meet criteria for MS and spinal cord MRI signal abnormality extending three or more vertebral segments termed longitudinally extensive spinal cord lesions (LESCL)), and one refers to CSF (pleocytosis $>50 \times 10^{6}$ white blood cells per L, or $>5 \times 10^{6}$ neutrophil per L).

Clinically isolated syndromes (CIS) such as AM and ON were diagnosed in those patients with monophasic AM or ON, without MRI features identified by meeting three of four Barkhof criteria, or by not showing at least two lesions plus the presence of OCBs in CSF. ${ }^{10}$

\section{Statistical analysis}

All statistical analysis (means, standard deviation), significance of group differences ( $\chi^{2}$ and T-tests) and correlation between variables were performed using the Statistical Program for Social Sciences (SPSS) statistical software (V12.0 for Windows). $P$ values of $<0.05$ were considered to be statistically significant.

\section{RESULTS}

This patient cohort was predominantly Caucasian, with $<1 \%$ being exclusively non-Caucasian. Existing epidemiological data suggests that the 842 cases represent approximately two-thirds of the entire MS population in WA, ${ }^{13-15}$ and that they comprise a representative sample of the total. There was a relatively low frequency of OSMS as a proportion of total demyelinating disease occurring in WA, with 31 OSMS (3.7\%) in contrast to 703 CMS (83.5\%) cases (fig 1). There were 11 OSMS cases who satisfied the diagnostic criteria for NMO. Of the remaining 108 patients, 50 had AM (5.9\%) and 42 had ON (5\%) as CIS and 16 were "atypical" cases (1.9\%) such as tumefactive MS, ADEM or were unable to be classified.
The mean age of onset in this present patient population was 36 years (SD 11.4, 12-78 years). The mean duration of disease was 12.5 years (SD 9.1, 0.5-55 years). There were 136 patients $(16.2 \%)$ who had been followed up for more than 20 years and the longest follow-up was 55 years. As a retrospective study, this MS cohort showed that the duration of disease follow-up inversely correlated with the age of onset $(r=-0.256$, $\mathrm{p}<0.001)$. The clinical phenotypic profile of this cohort remained consistent over the wide range of follow-up durations. There were no significant differences in the clinical characteristics such as gender ratio, rates of positive OCBs, abnormal VEPs, MRI brain or spine abnormalities, and presence of LESCLs between patients with follow-up durations of $\leqslant 10$ years and those of $>10$ years. The same consistency was observed between the groups with less and more than 15 years or 20 years follow-up durations, with the exception of a statistically significant higher VEP abnormality rate in the group with follow-up $>15$ years $(81.6 \%)$ than those $\leqslant 15$ years $(68.4 \%$, $\mathrm{p}=0.016)$.

At the time of assessment, the majority of the study patients (87.3\%) had an MRI of the brain, $61.8 \%$ had an MRI of the spine and $89.1 \%$ had either brain or spinal MRI scans. In total, 294 patients $(34.9 \%)$ and 347 patients (41.2\%) had lumbar puncture (LP) and VEP studies, respectively. Male patients or those who had normal MRI brain were more likely to have CSF analysis ( $p=0.043$ and $p=0.047$, respectively). Patients with OSMS or AM had a higher rate of CSF study than those diagnosed as CMS $(p<0.01)$. Those with OSMS or ON as a CIS had a higher VEP study rate compared with CMS patients $(p<0.01)$.

When CMS and OSMS were compared, CMS had a higher female to male ratio $(2.84: 1)$ than OSMS $(1.2: 1 ; p=0.019)$, OCBs were present in $66.4 \%$ of CMS and $29.4 \%$ of OSMS $(p=0.003)$ cases, and VEP abnormalities were present frequently in both CMS (71.4\%), and OSMS (85\%) cases. Brain MRI abnormalities were observed in $96.2 \%$ of CMS and $48.4 \%$ of OSMS individuals $(p<0.001$; table 1$)$. OSMS cases had a slightly higher but not statistically significant rate of spinal MRI abnormalities (92.6\%) than CMS (85\%). However, long spinal cord lesions were observed in $22.2 \%$ of OSMS cases and in $3.4 \%$ of CMS cases $(p<0.001)$. LESCLs were associated with an elevated CSF WCC ( $p=0.01 ; n=9$, mean 61.9 (SD 183)) when compared with other abnormal spinal MRI cases $(n=129,5.6$ (12.1)).

CSF specimens from OSMS patients contained significantly higher WCC than CMS patients (41.44 (129.12), range 0-550/ $\mathrm{mm}^{3}$ vs 5.28 (19.39), range $\left.0-250 / \mathrm{mm}^{3}, \mathrm{p}=0.001\right)$. A significantly elevated CSF protein level $(>1.0 \mathrm{~g} / \mathrm{L})$ was found in three CMS cases, one OSMS case and one atypical case. Those cases with elevated CSF protein level $(>1.0 \mathrm{~g} / \mathrm{L})$ or CSF pleocytosis (>50WCC) were all OCB negative (fig 2 ).

When CSF OCB-positive and -negative patients were compared, female predominance was slightly higher in OCBpositive patients than in OCB-negative patients, although it was not significant $(p=0.1$, table 2$)$. The age of onset in OCBpositive patients was younger than in OCB-negative patients $(p=0.005)$. The average protein level in CSF was higher in OCB-negative cases $(p=0.016)$. Average cell counts were similar in OCB-positive and -negative groups. The presence of $\mathrm{OCB}$ was significantly associated with the prevalence of brain MRI abnormalities, with $92.6 \%$ of OCB-positive cases having abnormal brain MRI in comparison with $71.8 \%$ of negative cases $(p<0.001)$. In OCB-positive cases, the frequencies of VEP abnormalities (70.4\%) and spinal MRI abnormalities (85\%, $\mathrm{p}=0.08)$ were higher than in OCB-negative cases $(58.5 \%$ and 


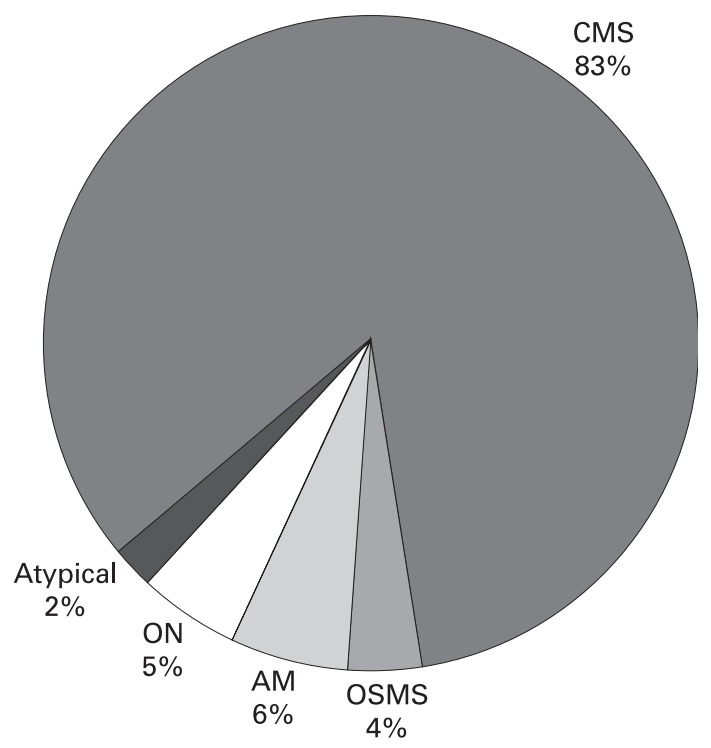

Figure 1 The spectrum of demyelinating disease in Western Australia. AM, acute myelitis; CMS, conventional form of multiple sclerosis; ON, optic neuritis; OSMS, optic-spinal multiple sclerosis.

$78 \%$, respectively). The association between the frequencies of OCBs and brain MRI abnormalities was also seen in the cohort of CMS, with $97.8 \%$ brain abnormalities being found in 137 OCB-positive patients in comparison with $91.4 \%$ in OCBnegative patients $(p=0.03)$. When the analysis was narrowed down to the CMS patients, the associations between presence of OCBs and increased frequencies of VEP abnormalities, spinal and brain MRI abnormalities were all statistically significant.

There were 113 patients with AM at presentation. Of these, $11 \mathrm{had}$ at least one further relapse of myelitis, 58 satisfied the diagnostic criteria of CMS, five did the same for OSMS, and 50 remained classified as AMCIS (acute myelitis clinical isolated syndromes). As a group, AMCIS patients had a similar male:female ratio as CMS patients, with an older average age at onset $(40.21(12.91))$ than CMS patients $(p=0.011)$. Of these AMCIS patients, 35 (79.5\%) had spinal MRI abnormalities and two had LESCLs. Five of 23 patients tested were OCB positive

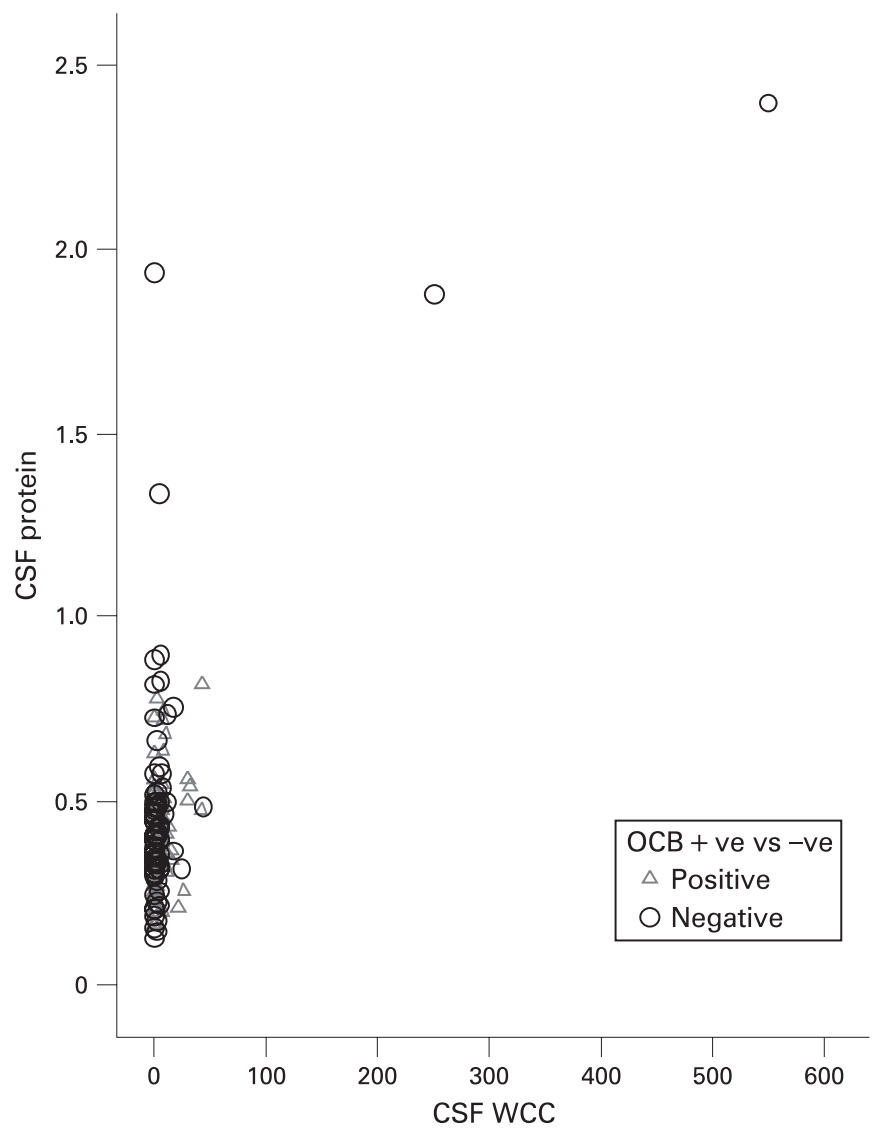

Figure 2 Cerebrospinal fluid (CSF) findings in patients with and without oligoclonal bands (OCBs). There were eight OCB-positive and 15 OCBnegative cases with elevated CSF protein level $(>0.6 \mathrm{~g} / \mathrm{L})$, and four cases with CSF protein higher than $1.0 \mathrm{~g} / \mathrm{L}$ were all OCB-negative. WCC, white cell count.

(21.7\%), five of 19 tested had abnormal VEPs (26.3\%), and six of 41 tested had brain MRI abnormalities (14.6\%).

The $42 \mathrm{ON}$ and $50 \mathrm{AM}$ patients had similar male:female ratios, durations of follow-up and CSF cell counts and protein levels to CMS patients. Of these ON patients, one was OCB positive. Not surprisingly, all 28 showed VEP abnormalities. A

Table 1 Clinical characteristics of demyelinating diseases in Western Australia

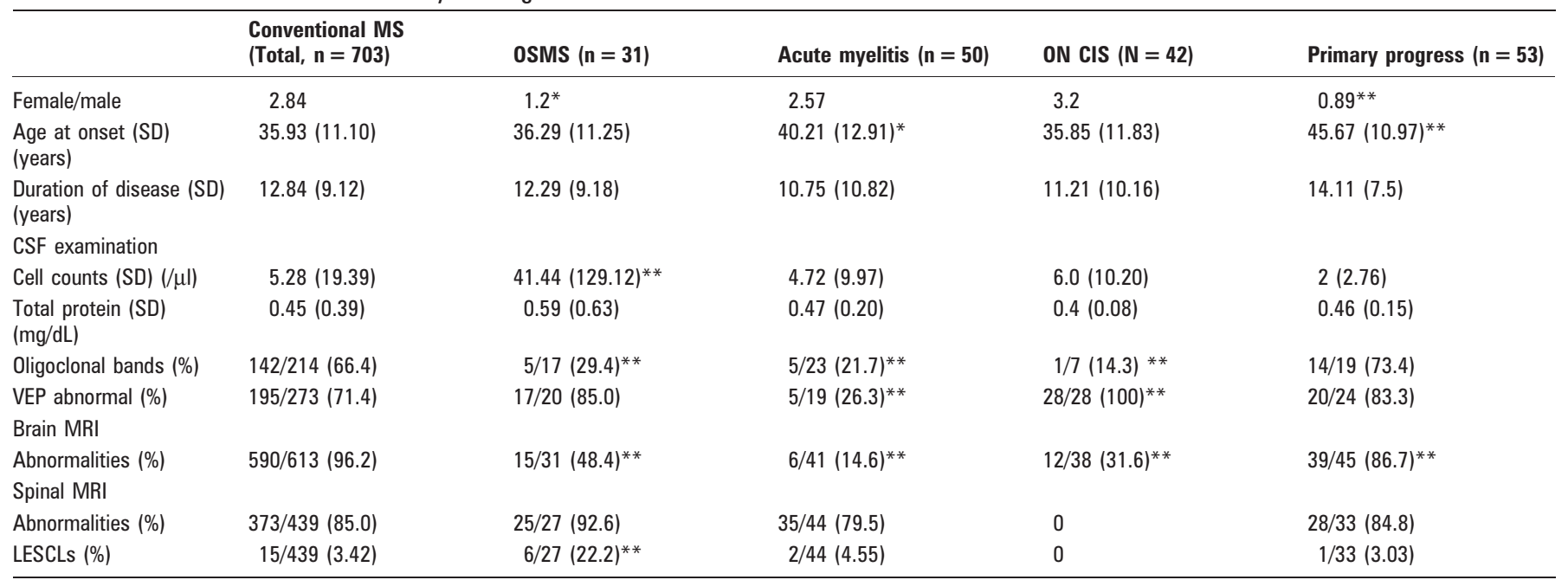

Values were significant in comparison with conventional MS: ${ }^{*} p<0.05$; ${ }^{* *} p<0.01$.

CSF, cerebrospinal fluid; LESCLs, longitudinally extensive spinal cord lesions; MRI, magnetic resonance imaging; MS, multiple sclerosis; ON CIS, optic neuritis clinically isolated syndromes; OSMS, optic-spinal multiple sclerosis; SD, standard deviation; VEP, visual evoked potential. 
Table 2 Comparison between clinical features of OCB and non-OCB patients

\begin{tabular}{lcc}
\hline & OCB & \\
\cline { 2 - 3 } & Positive $(\mathbf{n}=\mathbf{1 5 3})$ & Negative $(\mathbf{n}=\mathbf{1 1 9})$ \\
\hline Female/male ratio & 2.66 & 1.77 \\
Age at onset (SD) (years) & $35.35(10.82)^{* *}$ & $39.29(12.14)$ \\
CSF total protein (SD; $\mathrm{mg} / \mathrm{dL})$ & $0.40(0.14)^{*}$ & $0.54(0.55)$ \\
VEP abnormalities (\%) & $69 / 98(70.4)$ & $38 / 65(58.5)$ \\
Brain MRI abnormalities (\%) & $137 / 148(92.6)^{* *}$ & $79 / 110(71.8)$ \\
Spinal MRI abnormalities (\%) & $91 / 107(85)$ & $64 / 82(78)$ \\
\hline
\end{tabular}

Values were significant in comparison with negative OCBs: ${ }^{*} p<0.05 ;{ }^{* *} p<0.01$. CSF, cerebrospinal fluid; $\mathrm{OCB}$, oligoclonal band; MRI, magnetic resonance imaging; VEP, visual evoked potential.

total of $31.6 \%$ of ON patients had brain MRI abnormalities suggestive of MS. However, $35.7 \%$ of ON patients had insufficient follow-up to determine whether or not they met MS diagnostic criteria.

There was a total of 53 PPMS patients $(7.5 \%)$ with older age of onset (mean 45 years, $p<0.001$ ) and more frequent male gender (male: female ratio 1: $0.89, p<0.01$ ) compared to relapsing and remitting CMS (RRCMS) patients. There was no significant difference in CSF results and VEP findings between the PPMS and RRCMS patients. The OCB-positive rate in PPMS (73.4\%) was higher (but not significantly) than in CMS, whereas the prevalence of brain MRI abnormalities was slightly lower in PPMS (86.7\%) than in CMS patients $(96.2 \%$, $\mathrm{p}=0.004)$.

\section{DISCUSSION}

This cross-sectional retrospective study presents the full spectrum of demyelinating disease and gives a breakdown of the classification of MS in WA, largely preventing case selection and ascertainment bias that may have occurred in other studies. Australia has a well-funded and well-resourced National Health System with equal access to all members of society. Our study showed a relatively low frequency of OSMS (3.7\%) in this predominantly European-derived MS population. The clinical and investigational characteristics of OSMS in our study were consistent with reports of $\mathrm{MS}$ in Asia in many aspects: frequently elevated CSF cell counts, lower OCB-positive rate, less brain MRI involvement, and more common and severe spinal cord involvement compared to CMS. The exception was a lower female predominance in OSMS. ${ }^{216}$ The frequency of OSMS in this present study may be underestimated due to the fact that there were a significant number of potential OSMS cases who developed brain lesions in later life, despite initially having normal MRI brains or not satisfying the Barkhof criteria for CMS. Moreover, many were diagnosed before the availability of MRI so that the first MRI scans were performed some years after diagnosis. Thus, in this retrospective classification, such patients were classified as CMS rather than OSMS, whereas in Japanese studies the classification was made prospectively at presentation. ${ }^{2}$ A study from Amsterdam applied OSMS criteria and found that $8.8 \%$ in their cohort (124) were Caucasian patients with MS, results that were similar to those in Japan. ${ }^{17}$ We propose that differing interpretations of the criteria may explain many of the reported prevalence differences. Moreover, prospective data from newly diagnosed definite MS in Amsterdam found that 34\% did not meet Barkhof MRI criteria, and that 13 of 104 had long spinal lesions with a mean length of 11.4 segments. ${ }^{18}$ Interestingly, Asian studies have shown similar low rates of meeting Barkhof criteria. ${ }^{19}$ Parenthetically, there may be insufficient evidence to use failure to meet Barkhof MRI criteria to reclassify an MS patient with NMO or OSMS in either Western or Asian patients.

The vast majority of patients had MRI performed, because MRI is the investigation of choice and can be easily, rapidly and repeatedly accessed at no cost in Australia, with many patients having repeated MRI studies over time. As expected, VEPs were more likely to be performed in ON and OSMS, and CSF examination slightly more likely to be performed if brain MRI was normal (eg, AM or OSMS). These investigational patterns have not impacted on the results.

Our data showed that the current classification of CMS, OSMS or $\mathrm{NMO}, \mathrm{ON}$ and AM share many clinical features, such as age of onset, duration of disease and CSF protein and cell counts, suggesting that these presentations may be part of the spectrum or range of disease diversity. The MRI and CSF findings in CMS are also seen in ON, AM and OSMS cases. Distinguishing these groups from each other at the first presentation is not possible. Moreover, by strictly applying the current NMO diagnostic criteria to our MS patients, we found that there are many overlapping features: both CMS and OSMS can have the CSF and MRI abnormalities of the NMO supportive criteria (CSF pleocytosis and LESCLs). Not all NMO patients have severe attacks and poor prognosis. For example, there were three mild relapsing $\mathrm{NMO}$ patients presenting with bilateral relapsing $\mathrm{ON}$ and simultaneous AM. Four NMO patients had brain MRI abnormalities of the kind seen in CMS. In addition, patients with LESCLs have not necessarily had ON, and four LESCL patients had a normal VEP study. There were two CMS cases with typical LESCLs and positive OCBs. These heterogeneous clinical presentations suggest that the current diagnostic criteria and classifications are inadequate to encompass the full spectrum of neurological disease, and may give a false impression of the overall spectrum and behaviour of these illnesses.

It is frequently stated that OCBs are present in the majority of MS and, once detected, are permanently found. By comparison, OCBs are found uncommonly in $\mathrm{NMO}$ and, if present, can disappear in the course of the disease. ${ }^{20}$ The reported positive OCB rate in MS patients varies from more than $90 \%$ in European to $30-60 \%$ in Asian populations. ${ }^{2}$ The prevalence of positive OCBs in our patients with demyelinating disease was only $66.4 \%$ in CMS, $29.4 \%$ in OSMS, $21.7 \%$ in AM, $14.3 \%$ in ON and $73.4 \%$ in PPMS cases, which is comparable with reports on Asian MS. ${ }^{21}$ Our data was prospectively collected using sensitive validated isoelectric focusing methods and provides "real world" results for OCB positivity in diagnostic CSF examinations in MS. Our results are very different from the reported high positive rate from Europe, but virtually identical to the results in Japanese studies. This finding may have significant implications for the interpretation of CSF results in Western populations, and by extension for the application of Western CSF criteria in Asian populations.

This large population-based cohort of patients with MS has shown numerous similarities to prospectively studied cohorts from Asia, ${ }^{2}$ including low rates of $\mathrm{OCB}$ positivity. Clinical and laboratory features of the patients demonstrated a heterogeneous spectrum of disease, with no clear cut-off on any parameter between different disease patterns as they are currently defined. Detailed analysis of the long-term clinical assessments collected on this cohort should be possible and may determine whether or not disease patterns may have converged over time. Prospective testing of recently defined biomarkers of 
disease in MS, such as AOP4 IgG and HLA typing, will be interesting to compare with Asian reports, and may help to determine their clinical utility. At present, their role is uncertain. ${ }^{22-24}$

Acknowledgements: J-SW and M-NZ received financial support from BayerSchering, Biogen-Idec and Merck-Serono during this study.

Competing interests: None declared.

\section{REFERENCES}

1. de Seze J, Lebrun C, Stojkovic T, et al. Is Devic's neuromyelitis optica a separate disease? A comparative study with multiple sclerosis. Mult Scler 2003;9:521-5.

2. Kira J. Multiple sclerosis in the Japanese population. Lancet Neurol 2003;2:117-27.

3. Devic C. Myelite subaigue complique de nerrite optique. Bull Med 1894;35:18-30.

4. Wasay M, Khatri IA, Khealani B, et al. MS in Asian countries. Int MS J 2006;13:58-65.

5. Pittock SJ, Lennon VA, Krecke K, et al. Brain abnormalities in neuromyelitis optica. Arch Neurol 2006;63:390-6.

6. Walker RW, Keir G, Johnson MH, et al. A rapid method for detecting oligoclonal lgG in unconcentrated CSF, by agarose isoelectric focusing, transfer to cellulose nitrate and immunoperoxidase staining. J Neuroimmunol 1983;4:141-8.

7. Blumenhardt LD, Halliday AM. Hemisphere contributions to the composition of the pattern-evoked potential waveform. Exp Brain Res 1979;36:53-69.

8. Carroll W, Halliday A, Kriss A. Improvements in the accuracy of pattern evoked potentials in the diagnosis of visual pathway disease. Neuroophthalmology 1982;2:237-53.

9. Poser CM, Paty DW, Scheinberg L, et al. New diagnostic criteria for multiple sclerosis: guidelines for research protocols. Ann Neurol 1983;13:227-31.

10. Compston A, Edan G, Goodkin D, et al. Recommended diagnostic criteria for multiple sclerosis: guidelines from the International Panel on the diagnosis of multiple sclerosis. Ann Neurol 2001;50:121-7.
11. Kira J, Kanai T, Nishimura $Y$, et al. Western versus Asian types of multiple sclerosis: immunogenetically and clinically distinct disorders. Ann Neurol 1996;40:569-74.

12. Wingerchuk DM, Hogancamp WF, O'Brien PC, et al. The clinical course of neuromyelitis optica (Devic's syndrome). Neurology 1999;53:1107-14.

13. English D, de Wytt C, Maxwell IC, et al. The epidemiology of multiple sclerosis in three Australian cities: Perth, Newcastle and Hobart. Neurology 1988;38:980-6.

14. Simmons RD, Hall CA, Gleeson $\mathrm{P}$, et al. Prevalence survey of multiple sclerosis in the Australian Capital Territory. Herpes 2001;8:60-3.

15. Mana $\mathbf{P}$, Goodyear M, Chow AM, et al. Progressive increase in incidence and prevalence of multiple sclerosis in Newcastle, Australia: a 35-year study. $J$ Autoimmunity 2003;21:339-51.

16. Chang $\mathbf{K H}$, Lyu RK, Chen CM, et al. Clinical characteristics of multiple sclerosis in Taiwan: a cross-sectional study. Mult Scler 2006;12:501-6.

17. Eikelenboom M, Killestein J, Uitdehaag B, et al. Opticospinal multiple sclerosis: a pathogenetically distinct form? Mult Scler 2004;10:334-35.

18. Bot JC, Barkhof F, Polman CH, et al. Spinal cord abnormalities in recently diagnosed MS patients: added value of spinal MRI examination. Neurology 2004;62:226-33.

19. Chong HT, Ramli N, Lee KH, et al. Magnetic resonance imaging of Asians with multiple sclerosis was similar to that of the West. Can J Neurol Sci 2006;33:95-100.

20. Bergamaschi R, Tonietti S, Franciotta D, et al. Oligoclonal bands in Devic's neuromyelitis optica and multiple sclerosis: differences in repeated cerebrospinal fluid examinations. Mult Scler 2004;10:2-4.

21. Nakashima I, Fujihara K, Nagata T, et al. Oligoclonal IgG bands in Japanese multiple sclerosis patients. Tohoku J Exp Med 1999;189:259-65.

22. Chong HT, Kermode AG, Tan CT. The role of anti-aquaporin-4 antibody in Asian patients with multiple sclerosis: Confusions and controversies. Neurol Asia 2007; 12:135-9.

23. Wu JS, Matsushita T, Carroll WM, et al. Low sensitivity of aquaporin-4 antibody in multiple sclerosis, longitudinally extensive spinal cord lesions and neuromyelitis optica in Australians. Neurol Asia 2007;12:149-50.

24. Galetta SL, Bennett J. Neuromyelitis optica is a variant of multiple sclerosis. Arch Neurol 2007;64:901-3.

\section{Access all our original articles online even before they appear in a print issue!}

Online First is an exciting innovation that allows the latest clinical research papers to go from acceptance to your browser within days, keeping you at the cutting edge of medicine.

Simply follow the Online First link on the homepage and read the latest Online First articles that are available as unedited manuscripts in downloadable PDF form. The articles are peer reviewed, accepted for publication and indexed by PubMed but not yet included in a journal issue, so you'll be among the first to read them! 